\title{
Indicators of the appropriateness of long term prescribing in general practice in the United Kingdom: consensus development, face and content validity, feasibility, and reliability
}

\author{
Judith A Cantrill, Bonnie Sibbald, Stephen Buetow
}

\begin{abstract}
Objectives-To develop valid, reliable indicators of the appropriateness of long term prescribing in general practice medical records in the United Kingdom.

Design-A nominal group was used to identify potential indicators of appropriateness of prescribing. Their face and content validity were subsequently assessed in a two round Delphi exercise. Feasibility and reliability between raters were evaluated for the indicators for which consensus was reached and were suitable for application.

Participants-The nominal group comprised a disciplinary mix of nine opinion leaders and prominent academics in the field of prescribing. The Delphi panel was composed of 100 general practitioners and 100 community pharmacists.

Results-The nominal group resulted in 20 items which were refined to produce 34 statements for the Delphi exercise. Consensus was reached on 30, from which 13 indicators suitable for application were produced. These were applied by two independent raters to the records of 49 purposively sampled patients in one general practice. Nine indicators showed acceptable reliability between raters. Conclusions -9 indicators of prescribing appropriateness were produced suitable for application to the medical record of any patient on long term medication in United Kingdom general practice. Although the use of the medical record has limitations, this is currently the only available method to assess a patient's drug regimen in its entirety.
\end{abstract}

(Quality in Health Care 1998;7:130-135)

Keywords: prescribing; appropriateness; primary care

senior lecturer

Bonnie Sibbald, professor of health services

research

Stephen Buetow, research fellow

Correspondence to: Ms J A Cantrill,National Primary Care Research and Development Centre, 5th floor, Williamson Building, University of Manchester, Oxford Road, Manchester M13 9PL, UK. Telephone 00441612757601 ; fax 00441612757600 .

Accepted for publication 26 June 1998

Rising costs and demand for health care are prompting increased emphasis on the need to ensure quality and cost effectiveness in all aspects of care provision. Prescribing in United Kingdom general practice is no exception. General practitioner (GP) prescribing accounts for over $80 \%$ of the cost of all medicines, and $10 \%$ of the total NHS budget. This prescribing is often long term, with as many as three quarters of all prescriptions being for repeat items. The cost of medicines in
United Kingdom general practice is currently $>£ 4$ billion annually; a 1994 Audit Commission (box 1) report suggested that $£ 425$ million of this sum could potentially be saved through improvements in prescribing. ${ }^{1}$ However, we have argued elsewhere that the magnitude of the opportunities for cost savings is unclear. ${ }^{2}$ In France, one of the most recent attempts at cost containment has been the combination of mandatory practice guidelines on procedures and prescribing with a system of fines for doctors who do not comply. ${ }^{3}$ In the United Kingdom, various initiatives have been introduced nationally, including prescribing analysis and cost reports (PACT, box 1), the limited list, and professional prescribing advisors.

Prescribing analysis and cost data (PACT) were introduced in 1988 to enable GPs to look critically at their prescribing. PACT tells GPs what they have prescribed and how much their prescribing has cost in the preceding three month period. The data are produced by the Prescription Pricing Authority (PPA) and give information on both individual GPs and the practice's prescribing costs and compares these costs with those of other GPs in the same health authority (formerly, family health services authority) as well as nationally.

\section{The Audit Commission}

In 1990, the Audit Commission became responsible for the external audit of $\mathrm{Na}$ tional Health Service (NHS) bodies in England and Wales, including health authorities. The Commission's auditors are required to examine arrangements made by these authorities for securing economy, efficiency, and effectiveness in areas of expenditure for which they are responsible. Health authority responsibilities include oversight of primary care delivered by GPs and of dispensing by community pharmacists. Prescribing by GPs is the largest item in their budgets. 
patients' drug regimens. A more recent study has gone some way towards developing indicators to look more broadly at prescribing for individual patients. ${ }^{6}$ In this study, we have rejected the approach of deriving quality indicators from clinical guidelines as these are necessarily focused on specific conditions or drugs. Clinical guidelines focus on disease management, not patient management, and so may neglect interactions between treatment regimes in patients with two or more chronic conditions. We decided to base indicators on GP medical records, as opposed to other sources, because the medical record is presently the only source of routinely collected data that links prescribing with clinical information on individual patients. It is therefore the only existing database capable of allowing reviews of medication or assessments of appropriateness of prescribing. Appropriate health care has been defined by the RAND corporation as care which "means that the expected health benefit exceeds the negative consequences by a sufficiently wide margin that the procedure is worth doing". ${ }^{7}$ This has principally been applied to medical and surgical techniques in secondary care and has rarely considered prescribing. Publications in the United Kingdom refer more often to rational prescribing than to appropriate prescribing and the question of whether rational prescribing is necessarily the same as appropriate has rarely been considered. To assess appropriateness, we have advocated combining explicit criteria with expert review to allow implicit judgements to be made, thus enhancing the scope and richness of predetermined criteria. ${ }^{8}$ Most approaches to describing the appropriateness of prescribing have been based on poor or absent standards of reliability and validity. ${ }^{9}{ }^{10}$ The exception is the medication appropriateness index which exemplifies the use of explicit criteria to make implicit judgements of appropriateness. ${ }^{11}$ A recent, limited study in the United States which used the medication appropriateness index, suggested that inappropriate prescribing may be associated with increased use of the health service. ${ }^{12}$

Our aim is to develop indicators of the appropriateness of long term prescribing in general practice. An indicator has recently been defined by a European Working Party on quality in primary care (EQuiP) as: "A measurable element of practice performance for which there is evidence or consensus that it can be used to assess quality, and hence change in the quality, of care provided". ${ }^{13}$ In the first stage of our work, we convened a nominal group of experts to identify and describe dimensions of prescribing appropriateness. Subsequently, the face and content validity of the proposed dimensions were evaluated with the Delphi method in a panel of GPs and community pharmacists. Finally, we assessed the feasibility and reliability of the dimensions on which there was consensus and which were suitable for application. This programme of work was undertaken between June 1995 and April 1996.

\section{Methods}

IDENTIFICATION OF DIMENSIONS OF THE APPROPRIATENESS OF PRESCRIBING

The nominal group technique is a structured process for gathering information from a group of people who have expertise in a particular area (box 2). Its main purpose is to generate ideas, although the technique has been used to develop consensus. The group is nominal to the extent that it is highly controlled and discussion is normally restricted to the final stages of the group process.

(1) Formulation and presentation of the question

(2) Silent generation of ideas in writing

(3) Round robin feedback from group members to record each idea on charts visible to the whole group

(4) Group discussion of each idea in turn for clarification and evaluation

(5) Individual voting on priority ideas through rating, or as in this case, rank ordering

(6) If wished, feedback of results, further discussion, and rerating

\section{Box 2 Six stages of the nominal group technique.}

Individual people were invited to participate in a nominal group after the proposal by professionals and academics in the field of prescribing. Panellists were predominantly doctors or pharmacists as these are the two principal professional groups involved in managing long term prescribing. They comprised a mixture of people who were either researchers in the field of prescribing or who were actively working to improve prescribing in primary care. The group comprised nine people from different backgrounds: a medical director of a (former) family health services authority, an academic, a medical sociologist, a representative from the $\mathrm{Na}$ tional Health Service Executive (NHSE), a community pharmacist, a family health services authority pharmaceutical advisor, an academic GP, a hospital physician in care of the elderly, and a general practice principal. The moderator was an experienced group leader with no clinical or professional interest in long term prescribing (SB).

The group was asked to consider the question, "How would one screen GP medical records for (in)appropriate medication prescribing?". In the first phase of the nominal group, participants recorded silently and independently their ideas. In round robin fashion, one item was collected from each panellist. This process was repeated in successive rounds until no more ideas were forthcoming. When all the items had been collected there was a structured, serial discussion of each one. Discussions throughout the nominal group were audiotaped and later transcribed to facilitate subsequent examination of key issues, and to ensure that when developing the Delphi questionnaire the research team remained true to the views expressed by the nominal group. The panel then reviewed all the proposed items and undertook a process of refinement to 
reduce the items to a manageable number. In the last stage of the nominal group, each panellist was asked to select from the abridged list the 10 that he or she perceived to be most important and to rank them from 1 (most important) to 10 (least important).

FACE AND CONTENT VALIDITY AND

DEVELOPMENT OF CONSENSUS

The Delphi technique was used to assess face and content validity and to develop consensus. The Delphi process is a survey technique for decision making among isolated, anonymous respondents (box 3). It aims to guide opinion towards a final decision through feedback and reflection.

(1) Development and piloting of the questionnaire

(2) Establishment of definition(s) of consensus

(3) Identification and agreement of participants

(4) Posting the questionnaire and item rating by participants

(5) Individual feedback through provision of numerical data and summary of written comments from participants and rerating

Box 3 Stages of the Delphi technique.

The Delphi questionnaire was posted to a panel of community pharmacists and GPs. Eligible pharmacists were identified by the pharmaceutical advisors of family health services authorities who were asked for the names of community pharmacists actively working with GPs to improve prescribing. Eligible GPs were course organisers, appointed by regional postgraduate advisers in general practice to organise the day release element of vocational training for general practice. One hundred randomly selected professionals in each disciplinary group were sent a letter explaining the Delphi technique, the timescales involved, and an invitation to participate in the study. Those who did not reply or declined were replaced by another sample selected by the same method until the desired minimum sample of 100 of each discipline was attained.

The nominal group items were presented in the Delphi questionnaire as a series of statements, incorporating specific criteria by which each might be assessed.

Participants were asked to rate the "importance" of each item on a 7 point Likert scale ranging from $1=$ strongly disagree to $7=$ strongly agree (box 4). Space was provided for written comments beside each item. The questionnaire was piloted and refined in a local sample of five GPs and five community pharmacists.

The number of Delphi rounds was fixed at two at the outset. A summary of the findings from the first round was prepared in the subsequent two week period and fed back to participants. For each questionnaire item, this feedback comprised a bar chart showing the distribution of scores in the sample, the subject's own score, and a synopsis of the pan-

\begin{tabular}{|c|c|c|c|c|c|c|c|}
\hline \multirow{3}{*}{$\begin{array}{l}\text { Question } 7 \\
\text { (a) The dosing } \\
\text { frequency is } \\
\text { within the range } \\
\text { recommended } \\
\text { in the BNF } \\
\text { (b) If outside this } \\
\text { range, the } \\
\text { prescriber } \\
\text { records the } \\
\text { reason why }\end{array}$} & \multicolumn{2}{|c|}{$\begin{array}{l}\text { Of no } \\
\text { importance }\end{array}$} & \multicolumn{2}{|c|}{$\begin{array}{l}\text { Of some } \\
\text { importance }\end{array}$} & \multicolumn{2}{|c|}{$\begin{array}{l}\text { Of great } \\
\text { importance }\end{array}$} & \multirow{2}{*}{$\begin{array}{l}\begin{array}{c}\text { Com- } \\
\text { ment }\end{array} \\
7\end{array}$} \\
\hline & 1 & 2 & 3 & 4 & 5 & 6 & \\
\hline & 1 & 2 & 3 & 4 & 5 & 6 & 7 \\
\hline $\begin{array}{l}\text { For each it } \\
\text { from } 1 \text { to } 7 \text { tha } \\
\text { ation of its imp } \\
\text { you wish. For } \\
\text { your rating or }\end{array}$ & & $\begin{array}{l}\text { lea } \\
\text { cesp } \\
\text { ace. } \\
\text { ple, }\end{array}$ & & ccle & & $\begin{array}{l}\text { nun } \\
\text { dr ev } \\
\text { mm } \\
\text { orat }\end{array}$ & $\begin{array}{l}\text { nber } \\
\text { valu- } \\
\text { ents } \\
\text { e on }\end{array}$ \\
\hline
\end{tabular}

Box 4 Sample from the Delphi questionnaire

ellists' written comments. Participants were then asked to rerate each item and, as with the first posting, asked to return it within two weeks.

The following definitions of consensus were established before data analysis:

- Consensus that item should be retained: $\geqslant 75$ of participants scored the item $\geqslant 5$

- Consensus that item should be excluded: $\geqslant 75 \%$ subjects scored the item $\leqslant 3$

- No consensus: item which failed to meet either of the above criteria.

\section{FEASIBILITY}

We explored the production of workable definitions for all the dimensions for which consensus was reached. A pilot feasibility study was conducted by JC in a random sample of 25 patients on long term medication in one large practice in south Manchester. Patients requiring long term medication were defined as those prescribed one or more drugs, each of which was used on most days for a period of three or more months. Each indicator was presented as a statement offering a choice of four to six response categories. The response categories common to all indicators included whether or not the requisite information was recorded in the medical record; the recorded reason for a prescribing decision was valid; the indicator was applicable to that patient; and the rater was able to formulate an opinion based on the available information. The medical record was said to comprise both the computerised and the paper based notes maintained by the practice for each patient.

\section{RELIABILITY BETWEEN RATERS}

Levels of agreement between two independent pharmacist raters were assessed on all long term drugs prescribed for 49 patients in another large practice. In both practices samples were drawn by JC from the register of requests for repeat prescriptions. The sample for reliability testing was purposive, enriched with the types of inappropriate prescribing our instrument was designed to detect. The indicators were applied to a synopsis of the medical records produced by a member of the research team (JC). This approach was used to ensure that we were testing the reliability of the instru- 
Table 1 Criteria included in the Delphi questionnaire

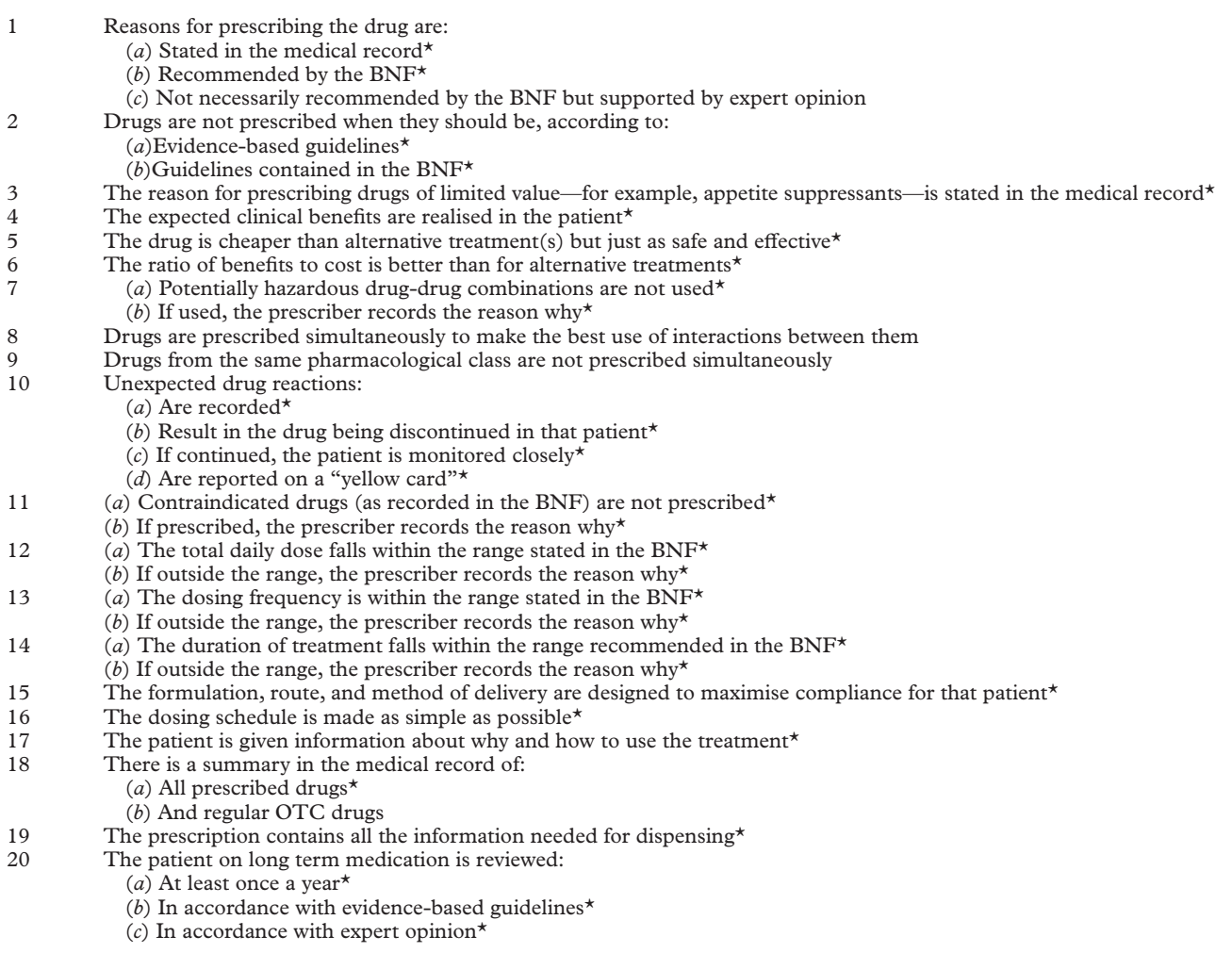

$\mathrm{BNF}=$ British National Formulary.

$\star$ Indicator retained after the Delphi exercise

ment, not the researchers' ability to find relevant information. Reliability was assessed by comparison of the response categories chosen by the two independent raters. We retained items where the $95 \%$ confidence interval included $\kappa \geqslant 0.7$.

\section{Results}

IDENTIFYING DIMENSIONS OF APPROPRIATENESS

OF PRESCRIBING

A total of 103 items was proposed by the panel and the final ranking process resulted in 20 items which were used to develop the 34 statements on the Delphi questionnaire (table 1).

\section{FACE AND CONTENT VALIDITY AND}

DEVELOPMENT OF CONSENSUS

More subjects was recruited than were required to meet our desired sample of $100 \mathrm{GPs}$ and 100 pharmacists. Of the 106 GPs who agreed to participate, 90 (85\%) completed round one and $66(62 \%)$ completed round two of the Delphi process. Of the 132 community pharmacists who agreed to participate, 108 (82\%) completed round one and $75(57 \%)$ completed round two. After round one, 27 of the 34 items met our consensus criteria for inclusion. Consensus was reached for a further three criteria after round two, giving a total of 30 items representing 18 potential indicators of prescribing appropriateness. The retained indicators are identified in table 1.

\section{FEASIBILITY}

We were unable to construct indicators suitable for application to medical records for six of the 18 dimensions (4, 6, 10,15, 17, 19; table 1). Suitable definitions were developed for the remaining indicators (table 2). Item 2 on the Delphi questionnaire was split into two separate indicators relating to hypertension and asthma (11 and 12; table 2), resulting in a total of 13 indicators. Alterations were made to the clarity of the questions and the response categories after feasibility testing.

Table 2 Operational indicators of prescribing appropriateness

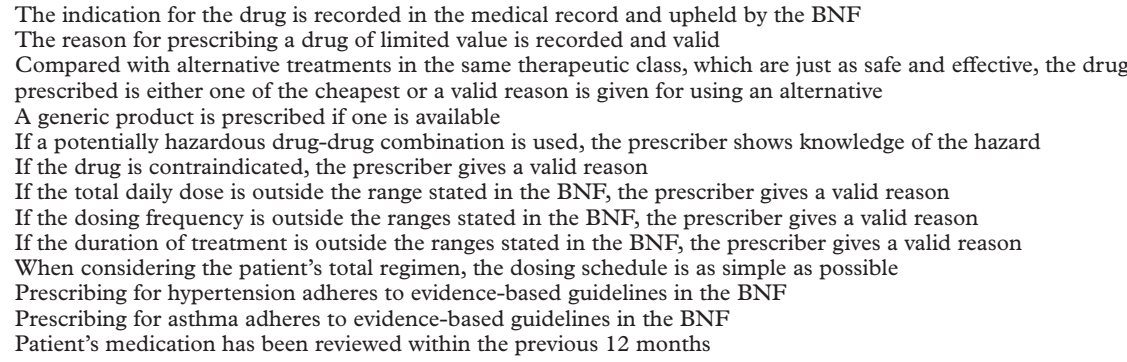


Table 3 Reliability between raters for indicators of prescribing appropriateness

\begin{tabular}{lllll}
\hline Indicator & $n$ & $\kappa$ & $95 \%$ CI & p Value \\
\hline Applied to individual drugs: & & & & \\
$\quad$ Indication recorded & 227 & 0.78 & 0.70 to 0.87 & $<0.0001$ \\
$\quad$ Reason if limited value & 227 & 0.96 & 0.89 to 1.04 & $<0.0001$ \\
Cost minimisation & 227 & 0.68 & 0.60 to 0.77 & $<0.0001$ \\
Generic & 227 & 0.89 & 0.83 to 0.95 & $<0.0001$ \\
Drug-drug interaction & 227 & 0.84 & 0.73 to 0.94 & $<0.0001$ \\
Dose & 227 & 0.54 & 0.38 to 0.70 & $<0.0001$ \\
Frequency & 227 & 0.61 & 0.43 to 0.79 & $<0.0001$ \\
Duration & 227 & 0.79 & 0.61 to 0.97 & $<0.0001$ \\
Contraindication & 227 & $\star$ & & \\
Applied to individual patient's records: & & & & \\
$\quad$ Overall dosing schedule & 49 & 0.34 & 0.10 to 0.58 & $<0.0035$ \\
$\quad$ Hypertension guidelines & 15 & 0.67 & 0.33 to 1.00 & $<0.0008$ \\
Asthma guidelines & 13 & 0.17 & -0.32 to 0.66 & $=0.246$ \\
Medication review & - & $\dagger$ & & \\
\hline
\end{tabular}

«Not adequately tested due to low prevalence.

†Not tested in study practice. put from the nominal group was 20 items which the Delphi questionnaire confirmed had face and content validity according to a selected group of GPs and community pharmacists. No item proposed by the nominal group was rejected by the Delphi panel. We do recognise that there are potential limitations to both methods, most notably the lack of robust evidence on their reliability and validity. ${ }^{14}$ However, our indicators are remarkably similar to those included in the medication appropriateness index. ${ }^{11}$ This close agreement between these two sets of indicators may suggest that the subject of inappropriate prescribing is not inherently contentious, as supported by the high levels of consensus achieved in the first round of our Delphi questionnaire.

Eleven of the 13 indicators used in the reliability study could be used to assess an entire drug regimen, as they are not disease or drug specific. Although we initially included two disease specific indicators (asthma and hypertension) we do not think that, when used in isolation, they give a broad enough picture of the (in)appropriateness of prescribing. Disease specific indicators are becoming widely accepted in clinical practice, particularly within the context of medical audit, and are relatively easy to apply. However, if clinical guidelines were to be the only source of prescribing indicators, a large proportion of long term prescribing would never come under scrutiny. Publications in the United Kingdom contain only a few examples of management guidelines for a limited range of diseases encountered in general practice. ${ }^{4}{ }^{15-17}$ It is interesting to note that although there are national guidelines for management of asthma in the United Kingdom, ${ }^{17}$ in this study we were not reliably able to apply them.

The PACT data are undoubtedly a valuable tool for examining and improving some aspects of general practice prescribing. However, a limitation of these data is their inability to link a prescription to a diagnosis. This becomes particularly relevant when a drug is indicated for more than one condition. For example, the ratio of inhaled steroids and cromoglycate to bronchodilators has been proposed as an indicator of performance in general practice that is available to Health Authorities. ${ }^{18}$ Although this indicator is arguably of value for judging the quality of care for asthma, it is inappropriate for chronic bronchitis, another prevalent disease in general practice. Bronchodilators are of symptomatic benefit for many patients with chronic bronchitis or emphysema, but inhaled steroids are rarely of value. Thus it may be argued that a high steroid:bronchodilator ratio represents poor prescribing in the context of non-asthmatic respiratory disease.

Although our goal is to produce indicators of appropriateness of long term prescribing which can be applied to an individual patient's drug regimen in its entirety, this approach does have some limitations. Use of medical records may invite criticism as some aspects of our indicators will rely on the level of documentation by the prescriber. In this study, there were several potential indicators with established face and 
content validity, which we were not able to apply, as we knew from our own experience that the pertinent information would not be found in the patient's record. However, there are precedents for assessing appropriateness through the data within medical records. In the United States, legislation allows deviation from prescribing guidelines for psychotrophic drugs only if the prescriber has documented a rationale for their use. ${ }^{19}$ Poor documentation may in itself represent poor quality patient care. ${ }^{20}$ However, it must also be acknowledged that this method of detailed data collection is currently resource intensive. The medication appropriateness index, which is also based on data in medical records, has been used both as a descriptive research tool and to measure outcomes from interventions aimed at improving prescribing. We also recognise that use of the medical records will rarely take into account the patient's perspective on prescribing. Supplementary measures are required if we wish to consider such issues as adherence to prescribed treatment, satisfaction with prescribing decisions, and quality of life. However, indicators based on medical records represent a step forward in the pursuit of methods to assess the quality of prescribing decisions. The continuing development of computer technology related to prescribing in general practice ${ }^{21}$-for example the electronic British National Formulary (eBNF), CAPSULE, and PRODIGY - may further facilitate the development of record based prescribing indicators. Throughout this work, the BNF has been used as the "gold standard" for applying many of our indicators. With the growing use of eBNF it is highly probable that they could, in the future, be applied electronically to general practice databases. Further, we anticipate that as the work develops, individual indicators will show different prevalences Those which show high prevalence could be used to target specific problems - for example, our work to date suggests that drugs are often prescribed without any recorded indication. This raises the potential to develop a tool box of prescribing quality measures which are applied with various methods. Some may be developed and measured centrally or locally with PACT data, alongside others which are derived by computerised application to the practice database, whereas others are applied manually to consider a specific issue in a sample or subpopulation. In the next stage of our work, we will assess the content validity of these indicators through interviews with GPs about patient specific inappropriate prescribing. We will also use this opportunity to explore GPs' views of these indicators as a practical tool for measuring quality.

In conclusion, these indicators are currently too labour intensive to be used as routine performance indicators, but have potential value as a research tool. However, they do examine dimensions of long term prescribing not available through any other tool and as information technology develops, they should become more accessible.

We thank Mrs Shirley Halliwell for her administrative support, Dr Chris Roberts for statistical advice, and Miss Janey Dowell and Mrs Cathy Holland for their work in the reliability phase of the study. The work was funded by the National Primary Care Research and Development Centre which is in turn supported by the Department of Health.

1 Audit Commission. A prescription for improvement: towards more rational prescribing in general practice. London: HMSO, 1994.

2 Buetow S, Sibbald B, Cantrill JA. Prevalence of potentially inappropriate long term prescribing in general practice in inappropriate long term prescrib

3 Durand-Zaleski I, Colin C, Blum-Boisgard C. An attempt to save money by using mandatory practice guidelines in France. BMF 1997;315:943-6.

4 Sever P, Beevers G, Bulpitt C, et al. Management guidelines in essential hypertension: report of the second working party of the British Hypertension Society. BMF 1993; 306: 983-7.

5 Bateman DN, Eccles M, Campbell M, et al. Setting standards of prescribing performance in primary care: use of a consensus group of general practitioners. $\mathrm{Br} \mathcal{f} \mathrm{Gen}$ Pract 1996;46:20-5.

6 Oborne CA, Batty GM, Maskrey V, et al. Development of prescribing indicators for elderly medical inpatients $\mathrm{Br} F$ Clin Pharmacol 19;43:91-7.

7 Kahn KL, Kosecoff J, Chassin MR, et al Measuring the clinical appropriateness of the use of a procedure. Can we do it? Medical Care 1988;26:415-22.

8 Buetow SA, Sibbald B, Cantrill JA, et al. Appropriateness in health care: application to prescribing. Soc Sci Med 1997;45:261-71.

9 Schmader K, Hanlon JT, Weinberger M, et al. Appropriateness of medication prescribing in ambulatory elderly patients. F Am Ger Soc 1994;42:1241-7.

10 Brook RH, Kamberg CJ, Mayer-Oakes A, et al Appropriateness of acute medical care for the elderly: an analysis of the literature. Health Policy 1990;14:225-42.

11 Hanlon JT, Schmader KE, Samsa GP, et al. A method for assessing drug therapy appropriateness. 7 Clin Epidemiol 992;45:1045-51.

12 Schmader KE, Hanlon JT, Landsman PB, et al. Inappropriate prescribing and health outcomes in elderly veteran outpatients. Ann Pharmacother 1997;31:529-33.

13 Lawrence M, Olesen F. Indicators of quality in health care. Eur 7 Gen Pract 1997;3:103-8.

14 Cantrill J, Sibbald B, Buetow S. The Delphi and nominal group techniques in health services research. International Fournal of Pharmacy Practice 1996;4:67-74.

15 Betteridge DJ, Dodson PM, Durrington PN, et al. Management of hyperlipidaemia: guidelines of the British Hyperlipidaemia Association. Postgrad Med f 1993;69:359-69.

16 Paykel ES, Priest RG. Recognition and management of depression in general practice: consensus statement. $B M \mathcal{F}$ 1992;305:1198-202.

17 British Asthma Guidelines Co-ordinating Committee. British guidelines on asthma management: 1995 review and position statement. Thorax 1997:52:S1-24.

18 Majeed FA, Voss S. Prescribing indicators for general practice. BMF 1995;311:209-210

19 Elon R, Pawlson LG. The impact of OBRA on medical practice within nursing facilities. F Am Geriatr Soc 1992;40: 958-63.

20 Liesenfield B, Heekeren H, Schade G, et al. Quality of documentation in medical records of diabetic patients. Int $\mathcal{F}$ Qual Health Care 1996;8:537-42.

21 Wyatt J, Walton R. Computer based prescribing. BMF 1995;311:1181-2. 